\title{
BMJ Open Clopidogrel preventive effect based on cytochrome P450 2C19 genotype in ischaemic stroke: protocol for multicentre observational study
}

\author{
Tae-Jin Song (D) , ${ }^{1}$ Jinkwon Kim, ${ }^{2}$ Sang Won Han, ${ }^{3}$ Young Dae Kim, ${ }^{4}$ \\ Jong Yun Lee, ${ }^{5}$ Seong Hwan Ahn, ${ }^{6}$ Hye Sun Lee, ${ }^{7}$ Yo Han Jung, ${ }^{8}$ \\ Kyung-Yul Lee (D) ${ }^{8}$
}

To cite: Song T-J, Kim J, Han SW, et al. Clopidogrel preventive effect based on cytochrome P450 2C19 genotype in ischaemic stroke: protocol for multicentre observational study. BMJ Open 2020;10:e038031. doi:10.1136/ bmjopen-2020-038031

- Prepublication history and additional material for this paper are available online. To view these files, please visit the journal online (http://dx.doi. org/10.1136/bmjopen-2020038031).

Received 25 February 2020

Revised 19 May 2020

Accepted 08 July 2020

Check for updates

(C) Author(s) (or their employer(s)) 2020. Re-use permitted under CC BY-NC. No commercial re-use. See rights and permissions. Published by BMJ.

For numbered affiliations see end of article.

Correspondence to

Dr Kyung-Yul Lee;

kylee@yuhs.ac

\section{ABSTRACT}

Introduction Clopidogrel is an antiplatelet agent that is widely used for the secondary prevention of cardiovascular and cerebrovascular events. The genotype of cytochrome P450 2C19 (CYP2C19) differentially affects the liver's metabolism of clopidogrel, which may influence the drug's response and efficacy for cardiovascular event prevention. In contrast to prior studies of patients with coronary artery diseases, little is known about whether the CYP2C19 genotype influences the preventive efficacy of clopidogrel in patients who had a stroke. We hypothesise that, among patients who had an acute ischaemic stroke who are prescribed clopidogrel, the patients with a loss-of-function CYP2C19 genotype (poor and intermediate metabolisers) may be at a higher risk of composite cardiovascular events than those who are non-carriers (extensive metabolisers).

Methods and analysis This prospective observational multicentre study was designed to determine whether composite cardiovascular events would differ among patients who had an ischaemic stroke prescribed clopidogrel according to CYP2C19 genotype (poor or intermediate vs extensive metabolisers). Inclusion criteria were patients who had an acute ischaemic stroke who underwent CYP2C19 genotype evaluation and received clopidogrel within 72 hours of stroke onset. The primary outcome is composite cardiovascular events (stroke, myocardial infarction, or cardiovascular death) within 6 months after acute ischaemic stroke between patients categorised as poor or intermediate metabolisers and those categorised as extensive metabolisers according to their CYP2C19 genotype.

Ethics and dissemination The Institutional Review Board of Severance Hospital, Yonsei University College of Medicine approved this study (3-2019-0195). We received study approval from the institutional review board of each participating hospital. We plan to disseminate our findings at relevant conferences and meetings and through peerreviewed journals.

Trial registration number NCT04072705.

\section{INTRODUCTION}

Clopidogrel is an antiplatelet agent used as a secondary prevention in patients who had an ischaemic stroke, coronary artery
Strengths and limitations of this study

- This study will include a large sample size of patients who had an acute ischaemic stroke who received clopidogrel within 3 days after stroke onset.

- It will address the controversy of clopidogrel resistance according to CYP2C19 genotype in the secondary prevention of acute ischaemic stroke.

- The *2, *3 alleles of the CYP2C19 gene are relatively common in Asian populations; thus, the study sample size can be reduced.

- Our findings may not be widely generalisable since the study participants will all be Asian.

disease and peripheral artery disease. ${ }^{1-4}$ Clopidogrel exhibits antithrombotic effects by inhibiting platelet aggregation via P2Y12 (an adenosine diphosphate receptor on the platelets) by active metabolites that have undergone metabolism in the liver. ${ }^{5}$ In association with the metabolic pathway, there has been concern about the problem of clopidogrel resistance. The administration of drugs including atorvastatin or omeprazole that are subjected to the same metabolic processes as clopidogrel may cause inhibitory interactions. $^{67}$

Moreover, studies have shown that the genotype of cytochrome P450 2C19 (CYP2C19), an enzyme involved in the liver metabolism of clopidogrel, affects drug responses and recurrence rates of cardiovascular disease. Among persons treated with clopidogrel, carriers of a loss-of-function CYP2C19allele (poor or intermediate metabolisers) had significantly lower levels of the active metabolite of clopidogrel, decreased platelet inhibition and a higher rate of major adverse cardiovascular events, including stent thrombosis, than did noncarriers (extensive metabolisers). ${ }^{8}$ In patients with acute myocardial infarction receiving 
clopidogrel, particularly those who received percutaneous coronary intervention, patients who were CYP2C19 loss-of-function allele carriers had a higher rate of subsequent cardiovascular events than those who were not. ${ }^{9}$ Among patients with transient ischaemic attack or minor stroke, the use of both aspirin and clopidogrel compared with aspirin alone reduced the risk of recurrent stroke only in the subgroup of patients who were loss-of-function CYP2C19 allele non-carriers. ${ }^{10}$ In patients who had a small subcortical stroke, intermediate or poor metabolisers of clopidogrel had higher odds of recurrent stroke than extensive metabolisers in western populations. ${ }^{11}$ However, these studies were post-hoc analyses of patients who had a minor stroke or lacunar stroke subtypes. Prospective trials to date using large sample sizes to determine whether major cardiovascular events, including stroke, according to CYP2C19 genotype in patients who had an acute ischaemic stroke are limited. ${ }^{12}$ In addition, studies of the association between the CYP2C19 genotype and future major cardiovascular events in patients who had a stroke are lacking in the real-world practice setting.

We hypothesise that, among patients who had an acute ischaemic stroke who are prescribed clopidogrel, those carrying the loss-of-function CYP2C19 allele (poor and intermediate metabolisers) will be at higher risk of composite cardiovascular events including stroke, myocardial infarction and cardiovascular death than non-carriers (extensive metabolisers). To prove our hypothesis, we will perform the multicentre Prospective observationaL study to evAluate the effecT of clopidogrel on the prEvention of major vascuLar events according to the gEnotype of cytochrome P450 $2 \mathrm{C} 19$ in ischemic stroke paTients (PLATELET) trial.

\section{METHODS AND ANALYSIS}

\section{Study aims}

We aim to provide evidence to support our hypothesis that patients with poor or intermediate metaboliser CYP2C19 genotypes are more likely to present with the occurrence of composite cardiovascular events, including stroke, myocardial infarction and cardiovascular death, than those with extensive metaboliser CYP2C19 genotypes among patients who had an acute ischaemic stroke who received clopidogrel. The aims of this study are as follows.

\section{Aim 1}

Evaluate any difference in the incidence of composite cardiovascular events (stroke, myocardial infarction and cardiovascular death) between patients categorised as poor or intermediate metabolisers and those categorised as extensive metabolisers according to their CYP2C19 genotype.

\section{Aim 2}

Evaluate any difference in the development of ischaemic stroke, transient ischaemic attack, myocardial infarction, revascularisation (cerebrovascular, coronary, aorta and peripheral artery), deterioration of early neurological symptoms, or modified Rankin scale score (0-2) at 3 months after the index stroke between poor or intermediate metabolisers and extensive metabolisers according to CYP2C19 genotype.

\section{Aim 3}

Evaluate any difference in the incidence of all-cause mortality and bleeding events between poor or intermediate metabolisers and extensive metabolisers according to CYP2C19 genotype.

\section{Subjects and dataset}

This prospective observational multicentre study is designed to determine whether there are differences in composite cardiovascular events (stroke, myocardial infarction and cardiovascular death) in patients who had an ischaemic stroke according to CYP2C19 genotype. We will include patients who had an acute ischaemic stroke who received clopidogrel $(75-300 \mathrm{mg})$ within 72 hours from stroke onset and underwent CYP2C19 genotype evaluation. Patients who meet the inclusion criteria will be included in the study after the researcher has fully explained the plan and obtained written consent from them and/or their kin. In this study, the timing of the index stroke will be based on the first appearance of neurological abnormalities associated with it. However, in cases in which a patient wakes up and discovers a neurological abnormality or when it is difficult to know the exact time at which the neurological symptoms first occurred, the last time that the patient was neurologically normal will be defined as the timing of the index stroke. Because this is an observational and not randomised study, there are no restrictions on clopidogrel administration mode. However, coadministration with antiplatelet agents other than aspirin and clopidogrel will be limited, and the clopidogrel will be maintained until the end of the study. Subjects will be visited at 1, 3 and 6 months after the start of clopidogrel to check for the occurrence of cardiovascular events and adverse reactions.

\section{Inclusion/exclusion criteria}

From September 2019 to December 2022, we will include patients who meet the following inclusion criteria: (1) received confirmation of ischaemic stroke on brain computed tomography (CT) or magnetic resonance image (MRI); (2) received clopidogrel within 72 hours after ischaemic stroke; (3) age older than 19 years; (4) having agreed to participate in the study within 7 days after ischaemic stroke; and (5) underwent CYP2C19 genotyping. We will exclude patients who meet the following exclusion criteria: (1) taking oral anticoagulants at the time of screening or scheduled to take medication within 6 months; (2) require administration of antiplatelet agents other than aspirin and clopidogrel; (3) history of taking clopidogrel within 1 week prior to the index ischaemic stroke; (4) scheduled for coronary angioplasty and stent insertion, coronary artery bypass grafting, carotid 
endarterectomy and cerebral artery stent implantation within 6 months; (5) diagnosis of severe concomitant disease with an expected life span of less than 2 years or a malignant tumour that has not been cured; (6) having participated in another drug trial within the previous 30 days; (7) having findings corresponding to high-risk factors among the causes of cardiac embolism according to Trial of Org 10172 in Acute Stroke Treatment (TOAST) classification ${ }^{13}$; and (8) expected to have difficulty participating in this study and continuing according to the researcher's judgement.

\section{Measures and observation variables}

Table 1 lists the information, variables and parameters that should be checked during screening, at the three follow-up visits, and during unexpected visits. We will collect medical information about the following variables and parameters. At screening, written consent; date written consent was provided; subject identification number; demographic information (date of birth, sex, height, weight, body mass index); smoking status (packs/ year); history of hypertension, diabetes, dyslipidaemia, atrial fibrillation, coronary artery disease (myocardial infarction, unstable angina, percutaneous coronary artery intervention and coronary artery bypass surgery), stroke (transient ischaemic attack, cerebral infarction, cerebral haemorrhage, stroke not distinguished between cerebral infarction and cerebral haemorrhage), or malignancy; and information about the index stroke (timing of index stroke, hospital visit date, and date of first dose of clopidogrel after index stroke) will be collected.

We will determine CYP2C19 genotypes $(* 1, * 2, * 3$ and $* 17$ alleles). We will then define extensive metabolisers as patients with $* 1 / * 1, * 1 / * 17, * 17 / * 17$ genotypes, intermediate metabolisers as patients with only one $* 2$ or $* 3$ genotypes $(* 1 / * 2, * 1 / * 3, * 2 / * 17$ and $* 3 / * 17)$, and poor metabolisers as patients with only $* 2$ or $* 3$ genotypes $(* 2 / * 2, * 2 / * 3$ and $* 3 / * 3) .{ }^{14}$ In our study, the $* 1$ CYP2C19 genotype will be applied if the patients do not test positive for any of the other alleles.

We will acquire vital signs and routine blood laboratory test results. Information on medication history and concomitant medication after the index stroke (antiplatelet, antihypertensive, oral hypoglycaemic agents, insulin or lipid-lowering agents) will be obtained. Moreover, data will be collected regarding any thrombolytic therapies received (intravenous and/or intra-arterial), dose of clopidogrel and/or aspirin, admission date, discharge date, discharge paths (other departments, selfdischarge, transferred to another hospital or death). We will also collect data on what kind of brain imaging, brain vessel imaging and cardiac tests are used to determine the TOAST classification.

\section{Outcome measures}

The primary outcome measure of our study will be a difference in the incidence of composite cardiovascular events (stroke, myocardial infarction or cardiovascular death) within 6 months after acute ischaemic stroke between patients categorised as poor or intermediate metabolisers and those categorised as extensive metabolisers according to their CYP2C19 genotype. After enrolment, patients will be followed up at 1 month (visit 1), 3 months (visit 2) and 6 months (visit 3).

Recurrent stroke is defined as newly developed neurological symptoms lasting at least 24 hours or symptoms lasting less than 24 hours with relevant lesions visible on brain imaging after the index stroke. ${ }^{16}$ Myocardial infarction is defined as clinical evidence of acute myocardial ischaemia and the detection of a rise and/or fall of cardiac troponin values with at least 1 value above the 99th percentile of the upper reference limit and at least one of the following: (1) symptoms of myocardial ischaemia; (2) new ischaemic electrocardiography changes; (3) development of pathological $Q$ waves; (4) imaging evidence of new loss of viable myocardium or new regional wall motion abnormality in a pattern consistent with an ischaemic aetiology; or (5) identification of a coronary thrombus on angiography. ${ }^{17}$ Cardiovascular death is defined as a case of fatal stroke, fatal myocardial infarction or sudden death not clearly identified as being of non-vascular cause.

The secondary outcome measure of our study is to investigate whether there is a difference in the development of ischaemic stroke, transient ischaemic attack without ischaemic lesions on brain imaging, myocardial infarction, revascularisation (cerebrovascular, coronary, aorta and peripheral artery), deterioration of early neurological symptoms (when the total National Institutes of Health Stroke Scale score increases by 2 or more within 7 days of symptom onset), or the ratio of modified Rankin scale 0-2 at 3 months after the index stroke between patients categorised as poor or intermediate metabolisers and those categorised as extensive metabolisers according to their CYP2C19 genotype.

The tertiary outcome, including safety outcome, if the presence or absence of a difference in the incidence of all-cause mortality, fatal bleeding, symptomatic cerebral haemorrhage, ocular bleeding, bleeding requiring more than two packs of whole blood or red blood cells, and bleeding requiring hospitalisation between patients categorised as poor or intermediate metabolisers and those categorised as extensive metabolisers according to their CYP2C19 genotype.

\section{Data management and oversight}

All outcomes generated during the study will be reported to the steering committee, which will review the reported events and confirm whether they meet the definition of outcomes. Data can be requested from each investigator for an accurate decision. In addition, a steering committee meeting will be held every 6 months to review the study progress and resolve any issues arising. Other occasional meetings may be held at the members' request.

The anonymised research database for this study will be administered by the independent clinical research 
Table 1 Protocol of Prospective observationaL study to evAluate the effecT of clopidogrel on the prEvention of major vascuLar events according to the gEnotype of cytochrome P450 2C19 in ischemic stroke paTients study

\section{Period}

\begin{tabular}{|c|c|c|c|c|}
\hline Visit & $\begin{array}{l}\text { Screening } \\
\text { visit }^{\star}\end{array}$ & Visit 1 & Visit 2 & $\begin{array}{l}\text { Visit } 3 \\
\text { (end of study) } \dagger\end{array}$ \\
\hline $\begin{array}{l}\text { Week } \\
\text { ( } \pm \text { visit window) }\end{array}$ & Day 1 & $\begin{array}{l}4 \text { weeks } \\
( \pm 14 \text { days) }\end{array}$ & $\begin{array}{l}12 \text { weeks } \\
\text { ( } \pm 28 \text { days) }\end{array}$ & $\begin{array}{l}24 \text { weeks } \\
\text { ( } \pm 28 \text { days) }\end{array}$ \\
\hline Inclusion/exclusion criteriał Informed consentł & O & & & \\
\hline Demographics§ & O & & & \\
\hline Vital signף & 0 & O & 0 & 0 \\
\hline Medical history** & O & & & \\
\hline Information of index stroke ${ }^{\star \star}$ & O & & & \\
\hline TOAST classification ${ }^{\star \star}$ & 0 & & & \\
\hline Laboratory findings †† & O & & & \\
\hline CYP2C19 genotype†† & 0 & & & \\
\hline$m R S^{* \star *}$ & 0 & $\mathrm{O}$ & 0 & 0 \\
\hline Determination of whether to continue the study or to leave & & $\mathrm{O}$ & $\mathrm{O}$ & O \\
\hline History of antiplatelet medication $† \dagger \dagger$ & O & O & O & O \\
\hline 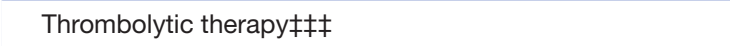 & $\mathrm{O}$ & & & \\
\hline Prior/concomitant medication§§§ & 0 & O & 0 & 0 \\
\hline Medical records of admission and discharge & $\mathrm{O}$ & & & \\
\hline Occurrence of outcomes & & $\mathrm{O}$ & $\mathrm{O}$ & $\mathrm{O}$ \\
\hline Adverse event ${ }^{\star * \star *}$ & & 0 & $\mathrm{O}$ & 0 \\
\hline
\end{tabular}

*Registration for the subject by confirming whether the final inclusion/exclusion criteria are met through the data collected at the screening visit.

†At case of early termination, we identify the reason for termination and perform the investigation as visit 3 .

$\ddagger$ A written informed consent will be obtained from the subject prior to starting this trial.

§Collection for date of birth, sex, height, weight and body mass index at the screening visit.

ๆVital signs will be collected each visit for blood pressure and pulse.

${ }^{*}$ We will check smoking history at screening, and then check smoking status at follow-up visits (visits 1-3). At screening, we will identify the history of the following diseases (hypertension, diabetes, hyperlipidaemia, atrial fibrillation, coronary artery disease (myocardial infarction, unstable angina, percutaneous coronary intervention, coronary artery bypass surgery), transient ischaemic attack, cerebral infarction, cerebral haemorrhage, stroke not identified with cerebral infarction/ haemorrhage and cancer). Stroke subtype will be investigated.

t+Laboratory test collection items are as follows (white cell count, neutrophil count, lymphocyte count, haemoglobin, haematocrit, platelet count, red blood cell distribution width, mean platelet volume, glucose (initial, fasting), blood urea nitrogen, creatinine, estimated glomerular filtration rate, liver panel, haemoglobin A1c, lipid panel, C-reactive protein, activated partial thromboplastin time, international normalised ratio, D-dimer, platelet drug response assay (VerifyNow P2Y12 reaction units test) and CYP2C19 genotype. Laboratory tests, CYP2C19 genotype and ECG collect the results of tests performed after index stroke.

$\ddagger \ddagger$ We check the results of brain image (MRI, magnetic resonance angiography, CT, computed tomography angiography and digital subtraction angiograph). Brain image data use results that are performed after index ischaemic stroke and before visit 1 .

$\S \S$ For evaluation of potential cardiac source of embolism, we will investigate and acquire the results of electrocardiography (ECG), cardiac echocardiography and Holter examination.

IIWe collect the National Institute of Health Stroke Scale (NIHSS) on every day during the hospitalisation period, from the date of hospitalisation to 7 days or to discharge day if patients discharge from hospital within 7 days.

${ }^{* \star *}$ At screening, we collect two types of modified Rankin scale (mRS), prestroke mRS (baseline mRS) and mRS at discharge. If the patient was discharged from visit 1 or later, the $\mathrm{mRS}$ at discharge was not required.

†† We will check whether antiplatelets are taken before prior index stroke and then the dose of clopidogrel, aspirin at follow-up visits.

$\ddagger \ddagger \ddagger$ At screening, we check for whether intravenous and/or intra-arterial thrombolysis was performed.

§§Information of prestroke medications will be collected only at screening. The collection criteria for prestroke and concomitant medications are as follows: prestroke medications: antiplatelet, oral anticoagulant, antihypertensive agents, oral diabetes mellitus medications, insulin and statins which are taken within 1 week prior to index stroke; concomitant medications: antiplatelet, antihypertensive agents, oral diabetes mellitus medications, insulin, statins and statins which are taken after index stroke.

10ाTThe admission record identifies the date of admission, the date of discharge and the route of discharge.

****In this study, only serious adverse events are collected.

CTA, computed tomography angiography; DSA, digital subtraction angiography; HbA1c, haemoglobin A1c; MRA, magnetic resonance angiography; TOAST, Trial of Org 10172 in Acute Stroke Treatment.

organiser. The study data will be stored on an encrypted website and only be accessible by the study researchers. Data monitoring will be independently performed by the sponsor without any competing interests. The site investigators will take responsibility for the data monitoring and conduct of the PLATELET trial. At each site, eligibility, protocol violations and participant outcomes will also be reported. Four clinical research associates 
are responsible for 36 institutions. Each site will be monitored at least twice including an initial visit by the clinical research associates. During the entire study period, a system query will occur using electronic case report forms and checked by the responsible clinical research associates. The site principal investigator will be responsible for documenting the occurrence of outcomes and reporting serious adverse events to the institutional review board of each site.

\section{Sample size and statistical power}

In the Clopidogrel versus Aspirin in Patients at Risk of Ischaemic Events study of patients who had recent ischaemic stroke, recent myocardial infarction or symptomatic peripheral arterial disease, the event rate per year of the composite outcomes including ischaemic stroke, myocardial infarction or cardiovascular death was 5.32\% among patients who took clopidogrel $(7.15 \%$ for the subgroup with recent ischaemic stroke). ${ }^{4}$ A meta-analysis comparing the risks according to the distribution of CYP2C19 genotypes in patients with acute cerebral infarction and transient ischaemia showed the risk of combined cardiovascular disease in the poor and intermediate metaboliser groups was about 1.51 times higher than that of the extensive metaboliser group. ${ }^{18}$ Considering the existing evidence in Korea regarding the distribution of CYP2C19 genotypes, it is estimated that poor and intermediate metabolisers account for $60 \%$ of the total population, while extensive metabolisers comprise the remaining $40 \%{ }^{19}$

Regarding our study's primary outcome, the incidence of composite cardiovascular events (stroke, myocardial infarction and cardiovascular death) during the 6 months after index stroke are set at $3 \%$ in the extensive metaboliser group and $4.5 \%$ in the poor and intermediate metaboliser groups. Considering the number of subjects needed to test the hypothesis that the event-free survival of both groups is the same at the 6-month follow-up using the log-rank test (two-tailed test, alpha $=0.05$ and 1-beta (power) $=0.80), 2634$ participants are required (1581 poor and intermediate metabolisers and 1053 extensive metabolisers). After the adjustment for an expected 10\% dropout rate, a total of 2927 subjects are required. Thus, this study will enrol a total of 2927 patients who had an ischaemic stroke that developed within 72 hours of the first symptom onset.

\section{Statistical analysis}

To summarise the data, we will demonstrate the descriptive statistics expressed as numbers with proportions for categorical variables and means with standard deviation or medians with interquartile ranges for continuous variables. To analyse the difference in baseline characteristics between the poor and intermediate metabolisers and the extensive metabolisers, the $\chi^{2}$ test, independent t-test and Mann-Whitney U-test are used for categorical variables, normally distributed continuous variables, and nonnormally distributed continuous variables, respectively.
The primary outcome of this study is the risk of developing composite cardiovascular events within 6 months. We will illustrate a Kaplan-Meier curve for the primary outcome and evaluate differences by CYP2C19 genotypes using the log-rank test.

To estimate the relative risk of each group, we will calculate the HRs and $95 \%$ CIs of the study outcomes using univariate and multivariate Cox proportional hazard regression models. In the multivariate model, adjustments will be made for sex, age and covariates (smoking, medical history, TOAST classification, National Institute of Health Stroke Scale (NIHSS) score, medication use and thrombolytic therapy) with values of $p<0.1$ in the univariate model. We will evaluate the differences in risks for the secondary and tertiary outcomes according to the CYP2C19 genotypes. Subgroup analyses will be performed by sex, individual CYP2C19 genotype, daily dose of clopidogrel, concurrent use of aspirin, initial stroke severity (NIHSS score) and TOAST classification.

\section{Patient and public involvement}

No patients or citizens were involved in the development of the research question. No patient advisers will be included in this research project.

\section{DISCUSSION}

The findings of studies on whether cardiovascular outcomes differ by CYP2C19 genotype in patients who had an stroke remain controversial. Previous studies demonstrated that reduced or lost CYP2C19 function was associated with poorer outcomes and an increased risk of recurrent stroke in patients who had an ischaemic stroke. ${ }^{20}{ }^{21}$ In contrast, no significant association of CYP2C19 status with future ischaemic events was found in patients who had an ischaemic stroke. ${ }^{22}$ In the overall cohort of the Secondary Prevention of Small Subcortical Strokes study, there were no differences in stroke recurrence according to CYP2C19 metaboliser status. ${ }^{11}$ In a meta-analysis, carriers of CYP2C19 loss-of-function alleles $(* 2, * 3$ and $* 8)$ were at a 1.92 -times increased risk of stroke compared with non-carriers. ${ }^{18}$ Composite vascular events were also more frequent (1.51 times higher) in carriers of CYP2C19 loss-of-function alleles than in noncarriers among patients who had an ischaemic stroke or transient ischaemic attack treated with clopidogrel. ${ }^{18}$ Our study will provide information about the risk of composite cardiovascular events, including stroke, myocardial infarction and cardiovascular death, in patients who had an ischaemic stroke treated with clopidogrel and elucidate whether there are differences in early neurological deterioration, functional outcomes and bleeding events according to CYP2C19 genotype.

Our study has some limitations. Because it is observational in nature, in the case of poor and intermediate metabolisers, the investigator can use dual antiplatelet therapy (aspirin plus clopidogrel) or modify the clopidogrel dose. Furthermore, it will be difficult to apply the 
results of the study to western populations because only Asian patients will be included. Nevertheless, its strengths are that CYP2C19 genotype effects will be examined in a large sample and that it will reflect the real-world practice of stroke treatment.

\section{Ethics and dissemination}

The Institutional Review Board of Severance Hospital, Yonsei University College of Medicine, approved this study (approval reference number: 3-2019-0195). We have registered the study at ClinicalTrials.gov (NCT04072705). Each institutional review board of the participating hospitals approved the study (online supplementary file 1 (ethics approval file) and online supplementary file 2 (informed consent)). We plan to disseminate our findings at relevant conferences and meetings and through peer-reviewed journals.

\section{Author affiliations}

${ }^{1}$ Department of Neurology, Seoul Hospital, College of Medicine, Ewha Womans University, Seoul, South Korea

${ }^{2}$ Department of Neurology, Yongin Severance Hospital, Yonsei University College of Medicine, Seoul, South Korea

${ }^{3}$ Department of Neurology, Inje University Sanggye Paik Hospital, Seoul, South Korea ${ }^{4}$ Department of Neurology, Severance Hospital, Yonsei University College of Medicine, Seoul, South Korea

${ }^{5}$ Department of Neurology, National Medical Center, Seoul, South Korea ${ }^{6}$ Department of Neurology, Chosun University Hospital, Gwangju, South Korea ${ }^{7}$ Biostatistics Collaboration Unit, Yonsei University College of Medicine, Seoul, South Korea

${ }^{8}$ Department of Neurology, Gangnam Severance Hospital, Yonsei University Collegel of Medicine, Seoul, South Korea

Contributors $\mathrm{K}-\mathrm{YL}$ is the $\mathrm{Pl}$ and led the writing of the grant application. HSL was responsible for the biostatistics analysis planning. T-JS and JK led the writing of the protocol manuscript. SWH, YDK, JYL, SHA and YHJ led the review and revision process of the protocol manuscript.

Funding This investigator-initiated clinical trial received funding support from Korea Samjin Pharmaceutical Co. (Seoul, Korea). The sponsor company is not involved in the study design or operations such as site selection and management, data management and manuscript drafting.

Competing interests None declared.

Patient and public involvement Patients and/or the public were not involved in the design, or conduct, or reporting, or dissemination plans of this research.

Patient consent for publication Not required.

Provenance and peer review Not commissioned; externally peer reviewed.

Open access This is an open access article distributed in accordance with the Creative Commons Attribution Non Commercial (CC BY-NC 4.0) license, which permits others to distribute, remix, adapt, build upon this work non-commercially, and license their derivative works on different terms, provided the original work is properly cited, appropriate credit is given, any changes made indicated, and the use is non-commercial. See: http://creativecommons.org/licenses/by-nc/4.0/.

\section{ORCID iDs}

Tae-Jin Song http://orcid.org/0000-0002-9937-762X
Kyung-Yul Lee http://orcid.org/0000-0001-5585-7739

\section{REFERENCES}

1 Hess CN, Hiatt WR. Antithrombotic therapy for peripheral artery disease in 2018. JAMA 2018;319:2329-30.

2 Yusuf S, Zhao F, Mehta SR, et al. Effects of clopidogrel in addition to aspirin in patients with acute coronary syndromes without STsegment elevation. N Engl J Med 2001;345:494-502.

3 Bhatt DL, Fox KAA, Hacke W, et al. Clopidogrel and aspirin versus aspirin alone for the prevention of atherothrombotic events. $N$ Engl J Med 2006;354:1706-17.

4 CAPRIE Steering Committee. A randomised, blinded, trial of clopidogrel versus aspirin in patients at risk of ischaemic events (CAPRIE). CAPRIE Steering Committee. Lancet 1996;348:1329-39.

5 Plosker GL, Lyseng-Williamson KA. Clopidogrel: a review of its use in the prevention of thrombosis. Drugs 2007;67:613-46.

6 Lau WC, Waskell LA, Watkins PB, et al. Atorvastatin reduces the ability of clopidogrel to inhibit platelet aggregation: a new drug-drug interaction. Circulation 2003;107:32-7.

7 Nguyen TA, Diodati JG, Pharand C. Resistance to clopidogrel: a review of the evidence. J Am Coll Cardiol 2005;45:1157-64.

8 Mega JL, Close SL, Wiviott SD, et al. Cytochrome P-450 polymorphisms and response to clopidogrel. N Engl J Med 2009;360:354-62.

9 Simon T, Verstuyft C, Mary-Krause M, et al. Genetic determinants of response to clopidogrel and cardiovascular events. N Engl J Med 2009;360:363-75.

10 Wang Y, Zhao X, Lin J, et al. Association between CYP2C19 lossof-function allele status and efficacy of clopidogrel for risk reduction among patients with minor stroke or transient ischemic attack. JAMA 2016;316:70-8

11 McDonough CW, McClure LA, Mitchell BD, et al. CYP2C19 metabolizer status and clopidogrel efficacy in the Secondary Prevention of Small Subcortical Strokes (SPS3) study. J Am Heart Assoc 2015;4:e001652.

12 Han SW, Kim Y-J, Ahn SH, et al. Effects of triflusal and clopidogrel on the secondary prevention of stroke based on cytochrome P450 2C19 genotyping. J Stroke 2017;19:356-64.

13 Adams HP, Bendixen BH, Kappelle LJ, et al. Classification of subtype of acute ischemic stroke. definitions for use in a multicenter clinical trial. TOAST. trial of ORG 10172 in acute stroke treatment. Stroke 1993;24:35-41.

14 Scott SA, Sangkuhl K, Stein CM, et al. Clinical Pharmacogenetics Implementation Consortium guidelines for CYP2C19 genotype and clopidogrel therapy: 2013 update. Clin Pharmacol Ther 2013;94:317-23.

15 Hicks JK, Sangkuhl K, Swen JJ, et al. Clinical pharmacogenetics implementation consortium guideline (CPIC) for CYP2D6 and CYP2C19 genotypes and dosing of tricyclic antidepressants: 2016 update. Clin Pharmacol Ther 2017;102:37-44.

16 Chang Y, Kim J, Kim Y-J, et al. Inter-arm blood pressure difference is associated with recurrent stroke in non-cardioembolic stroke patients. Sci Rep 2019;9:12758.

17 Thygesen K, Alpert JS, Jaffe AS, et al. Fourth universal definition of myocardial infarction (2018). Eur Heart J 2019;40:237-69.

18 Pan Y, Chen W, Xu Y, et al. Genetic polymorphisms and clopidogrel efficacy for acute ischemic stroke or transient ischemic attack: a systematic review and meta-analysis. Circulation 2017;135:21-33.

19 Jeong Y-H. Clopidogrel resistance. Korean J Med 2013;85:1-9.

20 Lin Y-J, Li J-W, Zhang M-J, et al. The association between CYP2C19 genotype and of in-stent restenosis among patients with vertebral artery stent treatment. CNS Neurosci Ther 2014;20:125-30.

21 Jia D-mei, Chen Z-bin, Zhang M-juan, et al. CYP2C19 polymorphisms and antiplatelet effects of clopidogrel in acute ischemic stroke in China. Stroke 2013;44:1717-9.

22 Qiu L-N, Sun Y, Wang L, et al. Influence of CYP2C19 polymorphisms on platelet reactivity and clinical outcomes in ischemic stroke patients treated with clopidogrel. Eur J Pharmacol 2015;747:29-35. 